\title{
PHYSIOLOGICAL LIMITATIONS ON PHYTOPLANKTON PRODUCTIVITY IN THE OCEAN
}

\author{
By Paul G. Falkowski, Richard M. Greene \\ and Richard J. Geider
}

$\mathrm{T}$

HE QUESTION of what limits primary productivity and phytoplankton biomass in natural waters has occupied oceanographers and limnologists for more than a century. The earliest oceanographers recognized that this issue is key to understanding the regulation of both aquatic food chains and biogeochemical cycles (reviewed by Mills, 1989). Traditionally, phytoplankton ecologists have been imbued with the concepts of the light, nitrogen and temperature limitation as they relate to the critical depth model (Sverdrup, 1953), Michaelis-Menten kinetics (Dugdale, 1967), and thermodynamic constraints on growth (Eppley, 1972), respectively. The recent suggestion that a micronutrient, such as iron, limits phytoplankton biomass or production in vast portions of the world's oceans (Martin, 1991) has been met with some skepticism (e.g., Banse, 1990). To a large extent the difficulty in determining what limits primary production stems both from methodological deficiencies and semantic confusion. The identification of limiting factors has been based on primarily three techniques: correlative inference, experimental manipulation, and simulation modeling. Here we examine the concepts of limiting factors and explore the possibility of using in vivo chlorophyll fluorescence (a biophysical signal), in conjunction with molecular markers, to identify or diagnose factors limiting phytoplankton growth and production in the ocean.

\section{Limiting Factors}

Historically, the meaning of the term "limiting factor" has been somewhat confusing in phytoplankton ecological literature. Two separate ecological concepts, both based on agricultural models, have emerged. The first, often called Liebig's Law of the Minimum, is a limitation of the standing stock by a substance that is least available relative to its requirement for biomass synthesis. The

P.G. Falkowski, R.M. Greene, Oceanographic and Atmospheric Sciences Division, Brookhaven National Laboratory, Upton, NY 11973, USA. R.J. Geider. College of Marine Studies, University of Delaware. Lewes, DE 19958, USA. qualitative applicability of this principle is immediately obvious in regional or basin-scale satellite maps of chlorophyll (Fig. 1); biomass is generally highest in areas where physical processes supply adequate nutrients (reviewed by Barber, 1992). Martin (1991), for example, assumed that iron limitation in the ocean follows Liebig's Law, i.e., that iron limitation is a constraint on phytoplankton biomass in certain regions. The second type of limitation, often attributed to the plant physiologist, F.F. Blackman (1905), relates the rate of growth to the availability of a nutrient. This type of limitation is much less obvious in the ocean (Cullen et al., 1992). Are high phytoplankton growth rates sustained (in oligotrophic regions of the ocean) by biological regeneration of nutrients (Goldman, 1980; Laws et al., 1987), or are the phytoplankton growing slowly because of physiological stresses imposed by an external limiting factor (Dugdale, 1967; Falkowski et al., 1991)? Distinguishing between the limitation of biomass and the limitation of growth rate is not merely a semantic issue. If only Liebig's Law applies, then the biomass-specific rate of primary production is nutrient independent and can be calculated readily from knowledge of only light and temperature. If, however, Blackman's concept applies, then nutrient-dependent physiological processes must be included in production models.

\section{The Concept of Diagnostics}

We use the term "diagnostics" to denote a signal (or procedure) that empirically identifies the symptoms of an environmental constraint on phytoplankton growth rates. The aim of diagnostics is to interrogate, without experimentally manipulating, natural phytoplankton. If, for example, the growth rate of cells is limited by temperature or the availability of nitrogen, iron, or light, then signals or markers induced by the limiting factor might provide a rapid, unambiguous means of identifying the limitation. Such markers could circumvent the need for long-term incubations in bottles (replete with all the attendant artifacts associated with that type of approach) supplemented with various suspected limiting factors. 


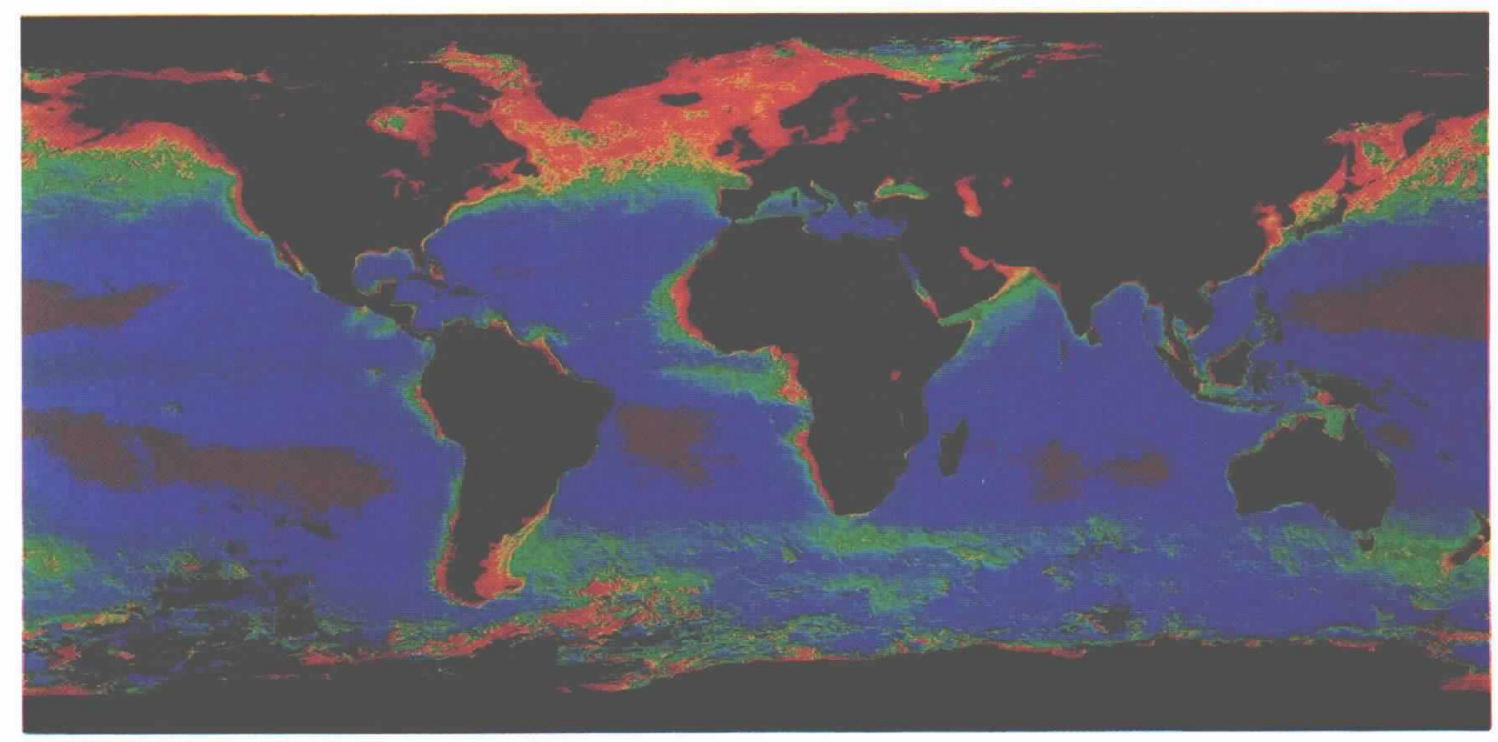

$\ldots$ if a nutrient is

physiologically limiting,

it will alter

photosynthetic

processes...

Fig. 1: The average annual distribution of chlorophyll in the upper ocean derived from composited Coastal Zone Color Scanner data from 1978 to 1986. This map reveals the relatively high concentrations of phytoplankton found in regions where nutrients are physically injected into the euphotic zone, such as in the North Atlantic, western North Pacific, the west coasts of Africa and South America, the equatorial Pacific, and the Antarctic Convergence (courtesy of Gene Feldman, Goddard Space Flight Center, National Aeronautics and Space Agency).

To be useful, diagnostic tools must identify those processes that 1) impose a truly physiological limitation, 2) are uniquely affected by a specific limiting factor, 3 ) are broadly applicable across phylogenetic lines, and 4) can be used in the field. Historically, the major approach to identifying limiting factors has focussed on quantitative analysis of photosynthesis-irradiance responses. This approach, borne largely because of the wide-scale accessibility of the radiocarbon method, is both logical and attractive. One of the major logical arguments for assaying the photosynthetic response is that the proteins that constitute the photosynthetic machinery or apparatus are highly conserved on both structural and functional levels in all oxygenic photosynthetic organisms (Raven, 1984; Falkowski, 1992), thus allowing inferences to be extended from the specific to the general. Additionally, the approach is attractive because chlorophyll can be easily used to operationally define phytoplankton and hence the photosynthetic biomass in natural waters. Moreover, as primary productivity is, to a first order, photosynthesis, the effect of limiting factors on the photosynthetic-irradiance response can be related to primary productivity.

An implicit assumption in the approach is that if a nutrient is physiologically limiting, it will alter photosynthetic processes from some non-nutrientlimited "normal" value. For example, Thomas (1970) inferred that phytoplankton in the eastern tropical Pacific were nitrogen limited, because the measured light-saturated rates of photosynthesis, normalized to chlorophyll $\left(\mathrm{P}_{\max }^{\mathrm{B}}\right)$, were lower than those found in more nitrogen-rich areas. Subse-
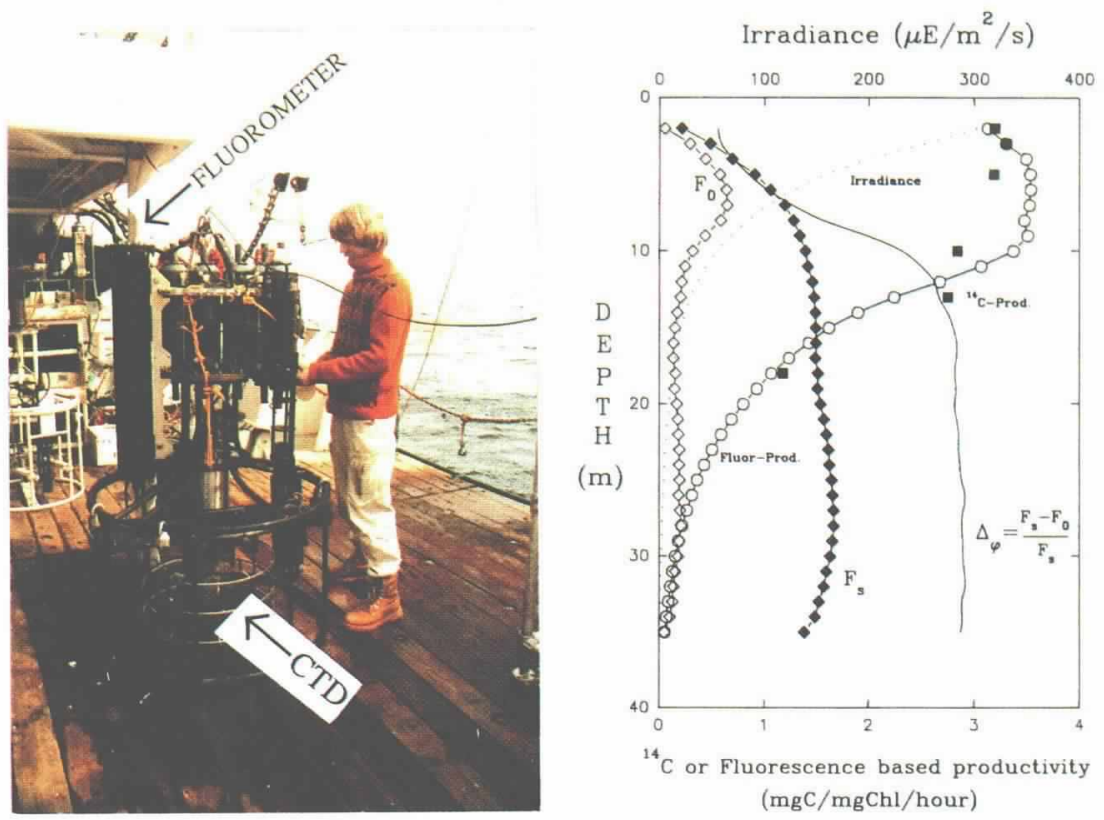

Fig. 2: Pump-and-probe fluorometer mounted on a rosette with a CTD. The instrument faces upward and allows samples to be assayed in ambient light. The right-hand panel shows a typical vertical profile of downwelling photosynthetically available radiation, $F_{0}, F_{s}$ and $\Delta \phi$ and compares the derived photosynthetic rates (Falkowski et al., 1991) with ${ }^{14}$ C-based estimates.

quent laboratory studies of photosynthetic characteristics for nitrogen-limited diatoms grown in chemostat cultures supported the interpretation of the field observations (Thomas and Dodson, 1972). Contradictory evidence was obtained in nitrogen-limited cultures by Eppley and Renger (1974), and only relatively small decreases in the 


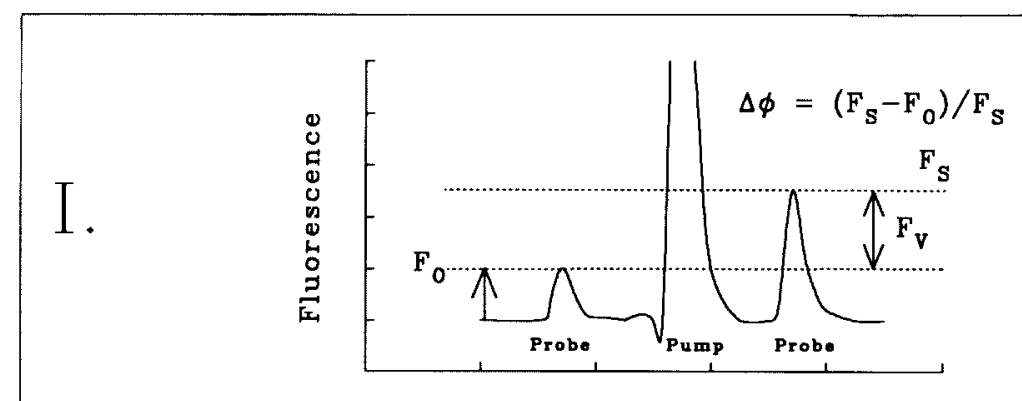

Time
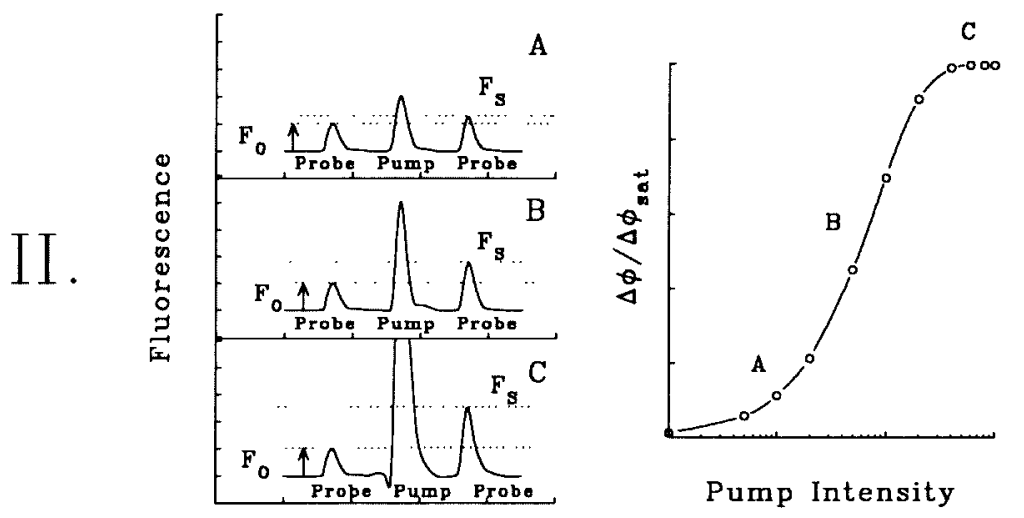

Time

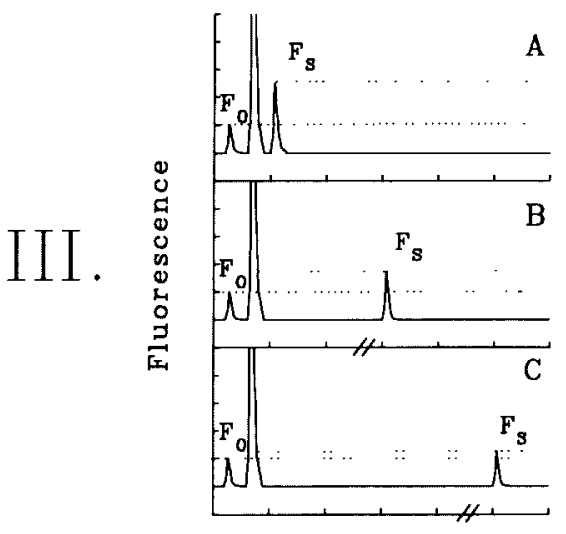

A

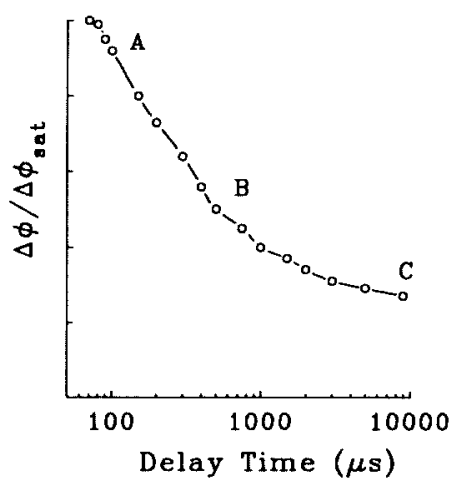

Time light-saturated photosynthetic rates have been measured in cultures (reviewed by Cullen et al., 1992). For oligotrophic waters, the historically measured values of radiocarbon assimilation appear to be suspect, because of trace metal contamination (Fitzwater et al., 1982). Therefore, one could legitimately ask whether the relatively small decreases in $\mathrm{P}_{\max }^{\mathrm{B}}$ values obtained by Thomas (1970) in nutrient-poor waters are related directly to nutrient limitation of photosynthesis or to trace-metal toxicity? Hence the use and interpretation of historical $\mathrm{P}_{\max }^{\mathrm{B}}$ data (with regards to nutrient limitation) is fraught with confusion.

Similar confusion attends the variability of the initial slope $(\alpha)$ of the photosynthesis-irradiance (P-I) curve in both laboratory and field studies (Platt et al., 1992). The initial slope of the P-I curve is the product of two independent parameters: the maximum quantum yield of photosynthesis and the optical absorption cross section of the ensemble of photosynthetic pigments. The quantum yield $(\phi)$ is the ratio of the number of molecules of carbon dioxide fixed to the number of photons absorbed. The optical absorption cross section is a measure of the average target area presented by chlorophyll and accessory pigments for photon absorption (Dubinsky et al., 1986). Until the mid $1980 \mathrm{~s}$, it was generally assumed that the optical absorption cross section was relatively constant (e.g., Kiefer and Mitchell, 1983); hence changes in $\alpha$ were implicitly assumed to reflect changes in quantum yield. However, laboratory studies (Bricaud et al., 1983; Falkowski et al., 1985) demonstrated that the absorption cross section normalized to chlorophyll could vary by a factor of about five, depending on growth conditions, increasing as cellular chlorophyll content decreases. Moreover, $\alpha$ appears to be a poor diagnostic, because increases in the optical absorption cross section are often accompanied by decreases in quantum yield. Subsequent field studies (Cleveland et al., 1989) implied that the optical absorption cross section varies significantly in natural phytoplankton communities; hence the derivation of the maximum quantum yield from the initial slope required a simultaneous measurement of the optical absorption cross section. Unfortunately, this parameter is not easily measured in field samples (Dubinsky, 1992).

Dark ${ }^{14} \mathrm{C}$ uptake was introduced as an index of nitrogen deficiency by Morris et al. in 1971 . The technique is based on the increase in $\beta$-carboxylation activity found in nitrogen-deficient cells in culture. The assay, which requires an incubation with and without added nitrogen, has been used to infer nitrogen limitation in natural waters. The dark ${ }^{14} \mathrm{C}$ incubation technique appears to be a relatively good diagnostic of nitrogen limitation, although the differences in dark carbon fixation between nitrogen-enriched and unenriched bottles may be relatively small in oligotrophic waters, even if cells are nitrogen limited. 
Table 1

Effect of light, nitrogen, and iron limitation on three fluorescence parameters.

\begin{tabular}{lccc}
\hline & \multicolumn{3}{c}{ Limiting Factor } \\
\cline { 2 - 4 } & Light & Nitrogen & Iron \\
\hline $\begin{array}{l}\text { Fluorescence parameter } \\
\text { Quantum yield }\left(\Delta \phi_{\text {sat }}\right)\end{array}$ & 0 & - & --- \\
$\begin{array}{l}\text { Absorption cross section } \\
\text { of PSII }(\sigma)\end{array}$ & ++ & ++ & ++ \\
$\begin{array}{l}\text { Turnover time of } \\
\text { electrons }\left(\tau_{1}\right)\end{array}$ & 0 & 0 & ++ \\
\hline
\end{tabular}

The effects. summarized from a number of specific laboratory studies, are indicated as increases $(+)$, decreases $(-)$, or no change $(0)$. The matrix suggests that by using a combination of fluorescence parameters, it is possible to diagnostically distinguish the physiologically limiting factors.

Many other diagnostic markers, symptomatic of nutrient, light, or temperature limitation have been proposed over the years. Such potential diagnostic markers as chlorophyll/cell, C/N (Goldman, 1980), $\mathrm{Fe} / \mathrm{C}$ (Anderson and Morel, 1982), and glutamate/glutamine (Flynn et al., 1989; Zehr et al., 1988) ratios, or the adenylate energy charge (Falkowski, 1977) can be related to specific limitations or stresses in laboratory cultures of phytoplankton. These markers are not easily applied to field studies, due either to the difficulty in physically separating phytoplankton from other particulate organic matter or to the high degree of interspecific variability in the signal. However, one readily measured signal, which is highly specific for phytoplankton, is related to photosynthesis, and yet is underexploited by biological oceanographers, is the in vivo fluorescence of chlorophyll introduced to oceanography by Lorenzen (1966).

\section{In vivo Fluorescence}

Assessing changes in the quantum yield for in vivo fluorescence of chlorophyll provides an alternate approach (to ${ }^{14} \mathrm{C}$ uptake) for investigating how photosynthetic processes are affected by suspected limiting factors. This technique is extremely attractive in that it requires little or no incubation or experimental manipulation, thereby largely avoiding problems of trace-metal toxicity and so-called bottle effects, and is specific for phytoplankton (Falkowski and Kiefer, 1985). The variation in the quantum yield of in vivo chlorophyll fluorescence can be quantitatively related to photosynthetic efficiency (Kiefer and Reynolds, 1992). Moreover, the maximum changes in the quantum yields of fluorescence are highly constrained by the evolutionary conservation of the photosynthetic machinery (Falkowski, 1992), thereby reducing interspecific or phylogenetic differences as a source of variability in the measurements.

We have investigated how fluorescence signals are affected by a variety of limiting factors in cul- tured phytoplankton and in natural phytoplankton communities. Our approach utilizes the pump-and-probe fluorescence technique (Falkowski et al., 1986), in which the change in the quantum yield of fluorescence $(\Delta \phi)$ for a weak "probe" flash is measured before $\left(F_{0}\right)$ and after $\left(F_{\mathrm{s}}\right)$ a stimulating (actinic) "pump" flash, $\Delta \phi=\left(F_{\mathrm{s}}\right.$ $\left.-F_{0}\right) / F_{0}$. The technique is rapid, nondestructive, and is amenable to an in situ vertical profiling system, such as a conductivity-temperature-depth (CTD) system (Fig. 2; Falkowski et al., 1991). Three basic parameters can be derived using the pump and probe method (see box); these include the maximum quantum yield of fluorescence $\left(\Delta \phi_{\text {sat }}\right)$, the absorption cross section of the photosynthetic machinery $(\sigma)$, and the rate of photosynthetic electron transport $(\tau)$.

We investigated how the three fluorescence parameters are influenced by light, nitrogen, and iron limitations. The results of studies with light and nitrogen limitation revealed that $\Delta \phi_{\text {sat }}$ is independent of growth irradiance, but is a hyperbolic function of specific growth rate, $\mu$, in nitrogen-limited cultures (Kolber et al., 1988). The relationship between $\Delta \phi_{\text {sat }}$ and the relative maximum specific growth rate, $\mu / \mu_{\max }$ (sensu Goldman, 1986), in nitrogen-limited cells could be modeled by a single curve, independent of species. Variability in $\Delta \phi_{\text {sat }}$ is not restricted to nitrogen limitation. We also found that $\Delta \phi_{\text {sat }}$ decreases as cells become increasingly limited by the availability of iron. The recovery from iron limitation is accompanied by an increase in $\Delta \phi_{\text {sat }}$, which, in the temperate diatom Phaeodactylum tricornutum and the Antarctic diatom Nitzschia curta, is correlated with an increase in the cellular abundance

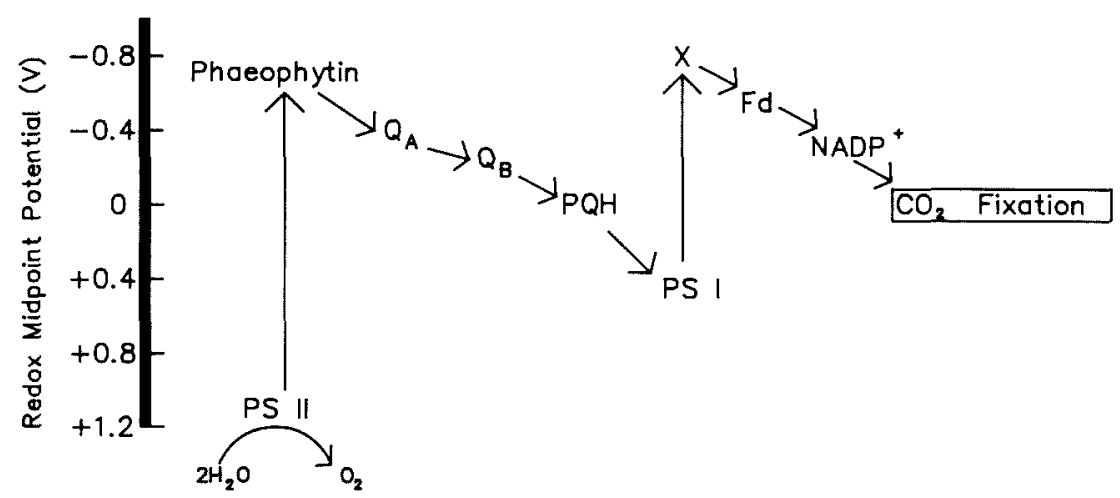

Fig. 3: Schematic diagram showing the intermediates involved in the initial stages of photochemistry for photosystem II. Upon absorbing the energy of a photon, a chlorophyll molecule in the PS II complex may briefly transfer an electron to phaeophytin and subsequently to a quinone, $Q_{A}$. This process occurs within a few hundred nanoseconds. $Q_{A}$ subsequently transfers its electron to a second quinone, $Q_{B}$; the half-time for this reaction is $\sim 160$ microseconds. The ratio of $Q_{A}^{-} / Q_{A}$ can be monitored by the pump and probe $\mathrm{flu}_{\mathrm{u}}$ orescence technique (see box) and can be quantitatively related to photosynthetic efficiency. Damage to or loss of reaction-center proteins (see Fig. 4) or the bound intermediates leads to a reduction in photosynthetic efficiency (Kolber et al., 1988). 
... one can potentially

distinguish between

light, nitrogen and iron

limitations. of chlorophyll. $\Delta \phi_{\text {sat }}$ is less sensitive to the availability of phosphate (R.J. Geider, J. LaRoche and R.M. Greene, unpublished observations). Thus, a change in $\Delta \phi_{\text {sat }}$ can be used to distinguish light from nutrient limitation (Table 1).

The absorption cross section of the photosynthetic machinery, $\sigma$, is also highly variable in phytoplankton. In light-limited cells, $\sigma$ generally (but not always, see Kolber et al., 1988) increases as cells acclimate to decreasing irradiance levels. However, $\sigma$ increases in both nitrogen- and ironlimited cells. This latter phenomenon appears somewhat counterintuitive. While both nitrogen and iron limitation lead to a decrease in cellular chlorophyll levels (in fact, chlorosis is a diagnostic of these limitations), the effective photon target size for the photosynthetic reactions increases (Kolber et al., 1988; Greene et al., 1991).

In both light- and nitrogen-limited cells, electrons are transferred out of the photosynthetic machinery with halftimes averaging $\sim 160 \mu \mathrm{s}$; however, in iron-limited cells this halftime increases to $\sim 500 \mu \mathrm{s}$. The increase in $\tau$ in ironlimited cells provides a biophysical diagnostic that differentiates between nitrogen and iron limitation (Table 1).

Thus, on the basis of fluorescence parameters alone, one can potentially distinguish between light, nitrogen and iron limitations (Table 1). These parameters are, with the proper equipment,

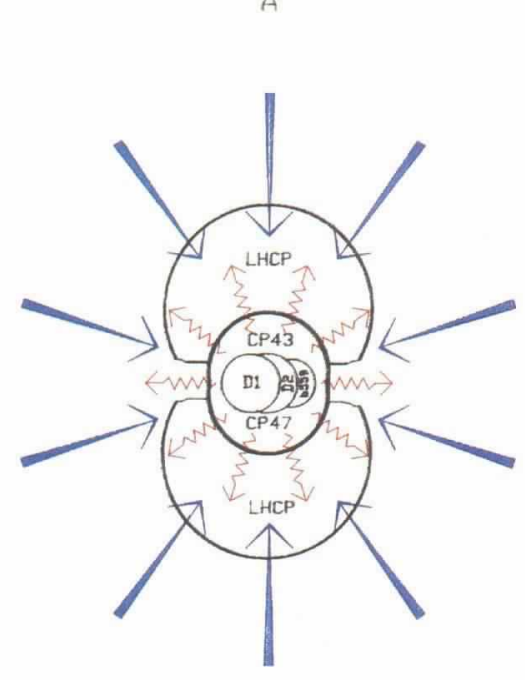

B

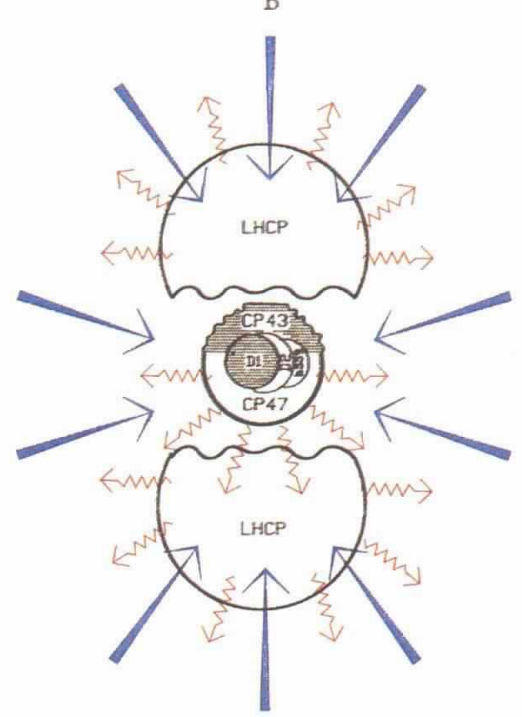

Fig. 4: Schematic diagram of photosystem II, showing the relationship of the distal antenna, light harvesting chlorophyll proteins (LHCPS) and the proximal antenna, CP 43 and $C P 47$, to the reaction-center proteins D1, D2 and cytochrome $b_{559}$. Under nutrient-replete conditions $(\boldsymbol{A})$, light energy (blue arrows) is absorbed by LHCP and transferred to the reaction center via the proximal antenna. About $2 \%$ of the absorbed energy is emitted as fluorescence (red arrows), primarily from the proximal antenna. Under nitrogen- and iron-limiting conditions $(\boldsymbol{B})$, there is a loss of DI and CP 43 in some reaction centers. Consequently, much of the absorbed light energy is not efficiently converted to photochemical energy, and a larger fraction (up to 5\%) is emitted as fluorescence. easily and rapidly measurable in natural phytoplankton communities without the need for manipulation or incubation. Although the fluorescence-based biophysical approach has many advantages, it is not extremely specific. For example, it may not be able to distinguish between nitrogen and iron limitation at low temperatures, where $\tau$ may be longer under both conditions. (Of course, if there is $25 \mu \mathrm{M}$ nitrate present, as in the case of many parts of the Southern Ocean or subarctic Pacific, it would be difficult to ascribe a simultaneous decrease in $\Delta \phi_{\text {sat }}$ and an increase in $\tau$ to nitrogen limitation.)

\section{Molecular Bases for Changes in Fluorescence Characteristics}

The fluorescence-based changes in nutrientlimited cells can be interpreted in terms of structural modifications (at the molecular level) to the photosynthetic machinery. In photosynthesis, the energy of light is used to extract electrons from water, producing oxygen, electrons and hydrogen ions. The electrons and hydrogen ions are used to chemically reduce carbon dioxide, thereby forming organic carbon. The photochemical conversions occur in two reaction centers, photosystem II (PS II), where the absorption of light leads to the oxidation of water, and subsequently in photosystem I (PS I), where the absorption of light leads to the reduction of intermediates that are used to fix carbon dioxide (Fig. 3). The two photosystems are connected by a variety of molecules that mediate the electron transfer between them. In living cells, virtually all of the fluorescence emanates from PS II.

The reaction center of PS II contains three proteins, D1, D2, and cytochrome $\mathrm{b}_{559}$ (Fig. 4). Excitation energy is delivered to the reaction center by two antenna systems, the proximal and distal antennae. The proximal antenna consists of two chlorophyll protein (CP) complexes, with molecular masses of 43 and 47 kilo Daltons, CP43 and CP47, respectively. These two chlorophyll proteins deliver excitation energy in parallel from the distal, light-harvesting chlorophyll protein complexes (LHCPs) to the reaction center. The LHCPs contain the bulk $(\sim 80 \%)$ of the photosynthetic pigments in the phytoplankton.

When PS II reaction centers are "open," excitation energy can be photochemically converted or "trapped" by passing electrons from a chlorophyll molecule through an intermediate phaeophytin molecule to an acceptor, $\mathrm{Q}_{\mathrm{A}}$. In the process, water becomes oxidized. The three molecules mediating the electron flow, chlorophyll, phaeophytin and $\mathrm{Q}_{\mathrm{A}}$ are all contained within the D1D2-cytochrome $b_{559}$ complex. If, however, $Q_{A}$ is already reduced by a previous excitation, the reaction center is said to be "closed," and the probability that excitation energy will be reemitted from CP43 and CP47 as fluorescence increases by a factor of $\sim 2.5$. 
Using polyclonal antibodies to specific proteins in the photosynthetic apparatus (reviewed by Ward, 1990), we have found that nitrogen and iron limitation lead to a loss of D1, CP43 and CP47 relative to LHCP in phytoplankton. The loss of these proteins decreases the efficiency for the transfer of excitation energy from LHCP to the reaction centers. The loss of D1 under nitrogen and iron limitation is correlated with a decrease in $\Delta \phi_{\text {sat }}$ (Kolber et al., 1988; Greene et al., 1991), indicating that under these conditions, photosynthesis per unit chlorophyll is reduced. The loss of reaction centers relative to LHCP simultaneously leads to an increase in the average photon target area for the reaction center; thus nitrogen- and iron-limited phytoplankton tend to become light saturated at lower photon flux densities.

\section{Other Molecular Markers}

While photosynthetic processes, as revealed by in vivo fluorescence and/or associated molecular diagnostic signals, appear to provide a means of assessing physiological limitations in natural phytoplankton communities, diagnostic markers are not limited to photosynthetic processes. Based on work with phytoplankton grown under controlled conditions in the laboratory, a number of modifications in proteins have been observed that appear to be potential indicators or diagnostics of specific limiting factors or stresses. For example, in marine cyanobacteria iron limitation leads to the induction of specific proteins in the cell membrane (Reithman and Sherman, 1988). Antibodies have been raised to some of these proteins and may be used to diagnose iron limitation in cyanobacteria in natural waters (N. Carr, personal communication). Similarly, in the marine diatom, $P$. tricornutum, a number of proteins appear to be altered by iron limitation (Fig. 5). Currently, in laboratories around the world, attempts are being made to isolate, purify and develop probes to detect molecular markers diagnostic of specific nutrient limitations. These probes would be used to supplement biophysical approaches, such as fluorescence.

\section{Evidence of Physiological Variability in Primary} Production in the Ocean

A central tenet that has emerged in biological oceanography over the past 50 years is that physical processes, by determining the distribution of nutrients and the mixed layer-depth, are primarily responsible for the distributions of primary producers and primary productivity on large time and space scales (Yentsch, 1980; Barber, 1992). This viewpoint relegates the importance of physiological processes in biogeochemical fluxes to small time and space domains (e.g., diel cycles). Is that viewpoint valid?

Using pump-and-probe fluorometry, we have found that the photosynthetic efficiency of natural phytoplankton communities varies by a factor of about two in the subtropical Pacific and Atlantic gyres and in the Gulf of Maine (Kolber et al., 1990; Falkowski et al., 1991). Similar methods employed to examine photosynthetic efficiency in the western boundary current and the Sargasso Sea and the northwest coast of Africa revealed a similar variation in quantum efficiency (Fig. 6). In all these studies, the quantum efficiency of photosynthesis is strongly, positively correlated with the availability of inorganic nitrogen.

Supportive, independent evidence that nutrient regimes affect the quantum yields in natural phytoplankton communities has recently emerged from simultaneous measurement of the initial slopes of photosynthesis-irradiance and deconvolution of in situ particulate absorption spectra (that are used to derive chlorophyll-specific absorption cross sections) (Cleveland et al., 1989; Bidigare et al., 1992; Platt et al., 1992). These methods suggest that the quantum yield of photosynthetic carbon fixation is lower in nutrient poor waters.

Further evidence that quantum yields are affected by nutrient availability is provided by calculation of the light utilization index, $\psi$ (Falkowski, 1981; Morel, 1991; Platt, 1986). This index is a composite of the average, column-integrated chlorophyll-specific absorption cross section and the average, column-integrated quantum yield (Morel, 1991). Data from a variety of nutrient-rich areas (mostly coastal regions) suggest that integrated primary production normalized to

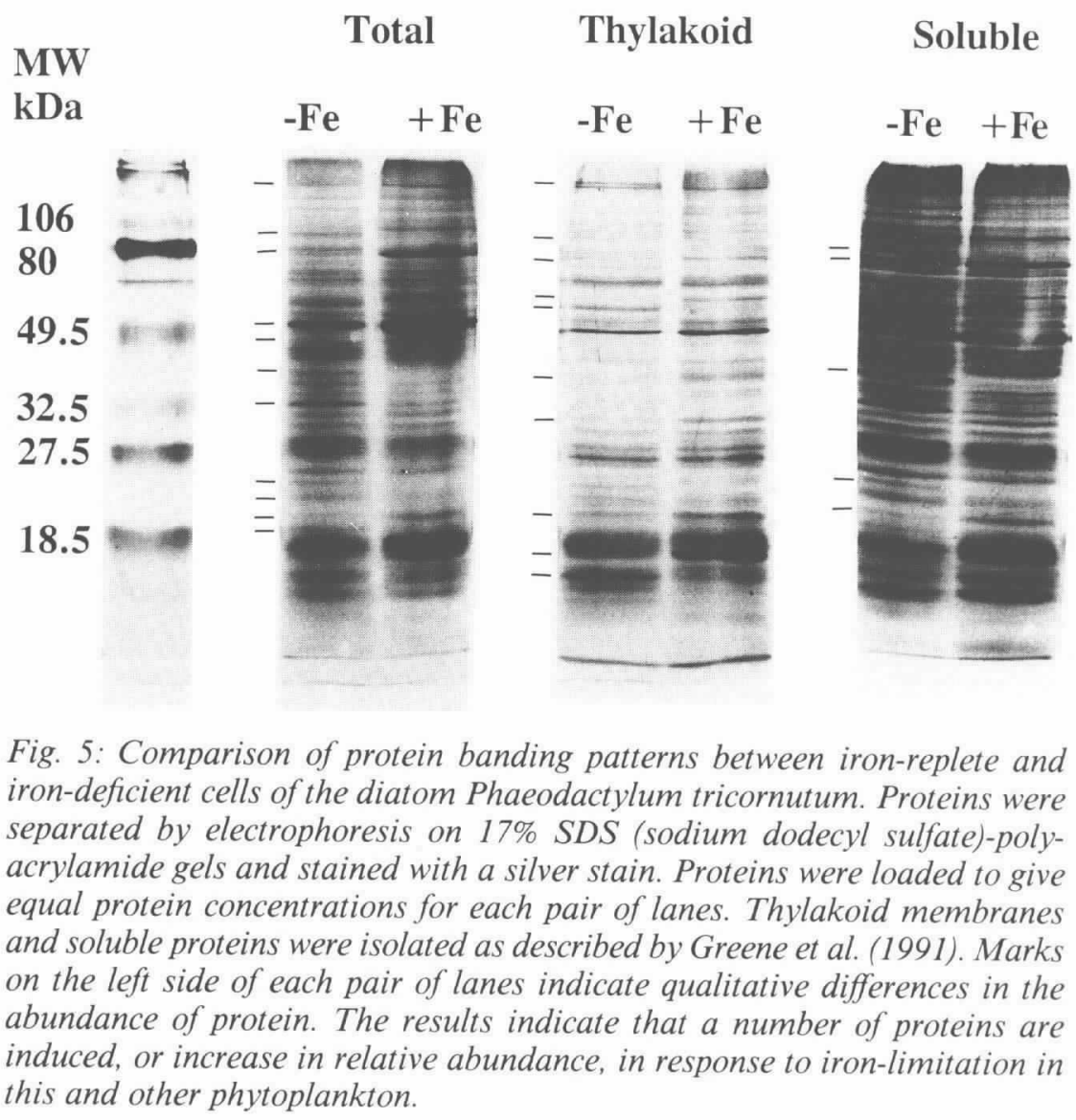

... the quantum

efficiency (of

photosynthesis) is

strongly positively

correlated with the

availability of inorganic

nitrogen. 
... in oligotrophic

regions of the ocean,

phytoplankton

photosynthesis, not

merely phytoplankton

biomass, is limited by

the availability of

nutrients. chlorophyll is linearly related to irradiance; hence, $\psi$ is highly constrained, averaging $\sim 0.44 \mathrm{gC}$ $\mathrm{gChl}^{-1}$ Einstein $^{-1} \mathrm{~m}^{-2}$. Data from oligotrophic regions show much more variability, however, and in many cases no linear relationship can be discerned. While part of the variability in $\psi$ for oligotrophic basins may be ascribed to variability in the chlorophyll-specific absorption cross sections (Morel, 1991), it appears that the variations in $\psi$ are larger than can be reasonably expected unless the quantum yield also varies.
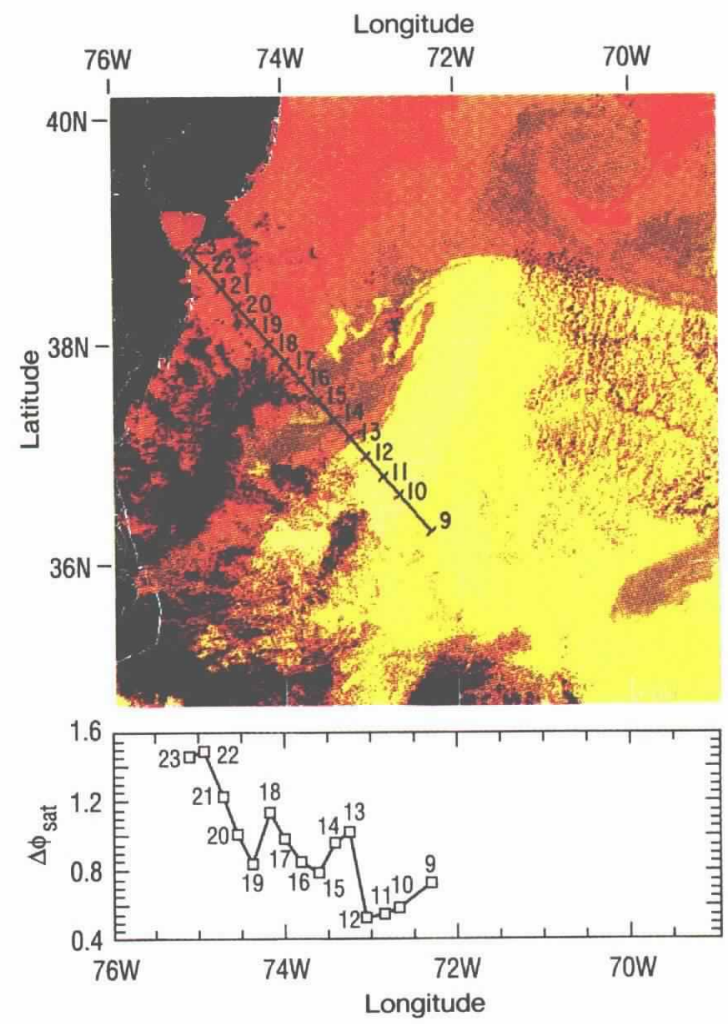

Fig. 6: An advanced very high-resolution radiometer (AVHRR) image showing sea surface temperature on October 8, 1991 extending from Cape Hatteras to the southern New Jersey coast. In this false-color representation higher temperatures are yellow, while cooler waters are orange and red. The line indicates a ship track taken between $O c$ tober 10 and 11 extending from Delaware Bay to the western Sargasso Sea. The station numbers are indicated. The ship traversed from the nutrientpoor Sargasso Sea, across the Gulf Stream, through a nutrient-rich filament of shelf water, across a region of nutrient-poor shelf-slope waters, and onto the continental shelf. The bottom panel shows the changes in variable fluorescence measured with a pump-and-probe fluorometer on the corresponding stations in near-surface natural phytoplankton communities. Note the two-fold variations in the changes in the quantum yields of fluorescence, which are primarily related to $n u$ trient availability in the given water masses (AVHRR image was provided by R. Evans, University of Miami, and P. Minnett, Brookhaven National Laboratory).
Taken together, the current evidence strongly suggests that in oligotrophic regions of the ocean, phytoplankton photosynthesis, not merely phytoplankton biomass, is limited by the availability of nutrients. These results contradict the hypothesis that the regeneration of nitrogen by the microbial loop is sufficient to maintain phytoplankton near their relative maximum specific growth rates (Goldman, 1980) and strongly suggest that the Blackman concept of rate limitation is applicable to phytoplankton in vast reaches of the ocean.

\section{Conclusions}

We have described a few of the techniques that have emerged over the past few years to allow biological oceanographers more direct examination of how external physical-chemical processes affect primary productivity. Our description is by no means comprehensive or complete, yet the initial applications of these techniques reveal that the efficiency with which natural phytoplankton communities convert solar radiation to stored chemical energy is highly dependent on nutrient supply. Information regarding regional and seasonal variability in quantum yields in the oceans is still lacking. Knowledge of such variability will be essential for using satellite data sets of ocean chlorophyll to estimate primary productivity. Nutrient-driven variations in the quantum yield imply that the most sophisticated time- and spaceresolved models of spectral irradiance distributions and knowledge of pigment concentrations always will be inadequate in calculating primary productivity, unless the physiological variability is explicitly parameterized. On an ecological level, the nutrient-dependent variability in quantum yields and other photosynthetic parameters suggests that growth rates, not merely crop size, are regulated by nutrient availability in the ocean.

\section{Acknowledgements}

This research was supported by the U.S. Department of Energy under Contract No. DEAC02-76CH00016 and NASA Grant UPN 16135-05-08, and EPA Grant IAG DW 8993523901-0 to P. Falkowski, and NSF grant OCG 8915084 to R. Geider. We thank Kevin Wyman, Julie LaRoche, Paul Kemp, Richard Barber and an anonymous reviewer for comments and criticisms. We also thank Gene Feldman, Bob Evans and Peter Minnett for help with satellite data representation.

\section{References}

Anderson, M.A. and F.M.M. Morel, 1982: The influence of aqueous iron chemistry on the uptake of iron by the coastal diatom Thalassiosira weissflogii. Limnol. Oceanogr., 27, 789-813.

Banse, K., 1990: Does iron really limit phytoplankton production in the offshore subarctic Pacific? Limnol. Oceanogr., 35, 772-775.

Barber, R.T., 1992: Geological and climatic time scales of nutrient availability. In: Primary Productivity and Biogeochemical Cycles in the Sea. P.G. Falkowski and A. Woodhead, eds., Plenum Press, New York, 89-106. 
Bidigare, R.R., B.B. Prezelin and R.C. Smith, 1992: Bio-optical models and the problems of scaling. In: Primary Productivity and Biogeochemical Cycles in the Sea. P.G. Falkowski and A. Woodhead, eds., Plenum Press, New York, 175-212.

Blackman, F.F., 1905; Optima and limiting factors. Ann. Bot., 19, 281-298.

Bricaud. A., A. Morel and L. Prieur, 1983: Optical efficiency factors of some phytoplankters. Limnol. Oceanogr.. 28 , 816-832.

Cleveland, J.S., M.J. Perry, D.A. Kiefer and M.C. Talbot. 1989 : Maximal quantum yield of photosynthesis in the northwestern Sargasso Sea. J. Mar. Res., 47, 869-892.

Crofts, A.R. and C.A. Wraight, 1983: The electrochemical domain of photosynthesis. Biochim. Biophys. Acta, 726. 149-185.

Cullen, J.J., X. Yang and H.L. MacIntyre, 1992: Nutrient limitation of marine photosynthesis. In: Primary Productivity and Biogeochemical Cycles in the Sea. P.G. Falkowski and A. Woodhead. eds., Plenum Press, New York, 69-88.

Dubinsky, Z., 1992: The functional and optical cross sections of phytoplankton photosynthesis. In: Primary Producivity and Biogeochemical Cycles in the Sea. P.G. Falkowski and A. Woodhead. eds., Plenum Press. New York, 31-46.

, P. Falkowski and K. Wyman, 1986: Light harvesting and utilizàtion in phytoplankton. Plant Cell Physiol. 27. 1335-1349.

Dugdale. R.C.. 1967: Nutrient limitation in the sea: dynamics, identification and significance. Limnol. Oceanogr., 12, 685-695.

Eppley, R.W., 1972: Temperature and phytoplankton growth in the sea. Fish. Bull., 70, 1063-1085.

and E.H. Renger, 1974: Nitrogen assimilation of an oceanic diatom in nitrogen-limited continuous culture. J. Phycol., 10, 15-23.

Falkowski, P.G., 1977: The adenylate energy charge in marine phytoplankton: The effect of temperature on the physiological state of Skeletonema costatum (Grev.) Cleve. J. Exp. Mar. Biol. Ecol., 27, 37-45.

1981: Light-shade adaptation and assimilation numbers. J. Plankton Res., 3, 203-206.

1992: Molecular ecology of phytoplankton photosynthesis. In: Primary Productivity and Biogeochemical Cycles in the Sea. P.G. Falkowski and A. Woodhead, eds., Plenum Press, New York, 47-68.

, Z. Dubinsky and K. Wyman, 1985: Growth-irradiance relationships in phytoplankton. Limnol. Oceanogr., 30, 311-321.

- and D.A. Kiefer, 1985: Chlorophyll $a$ fluorescence in phytoplankton: relationship to photosynthesis and biomass. J. Plankton Res., 7, 715-731.

K. Wyman, A. Ley and D. Mauzerall, 1986: Relationship of steady state photosynthesis to fluorescence in eucaryotic algae. Biochim. Biophys. Acta, 849, 183192.

D. Ziemann, Z. Kolber and P.K. Bienfang, 1991: Nutrient pumping and phytoplankton response in a subtropical mesoscale eddy. Nature, 352, 55-58.

Fitzwater, S.E. G.A. Knauer and J.H. Martin, 1982: Metal contamination and its effect on primary production measurements. Limnol. Oceanogr., 27. 544-551.

Flynn, K.J., D.M.J. Dickson and O.A. Al-Amoudi. 1989: The ratio of glutamate:glutamine in microalgae: a biomarker for $\mathrm{N}$-status suitable for use at natural cell densities. $J$. Plankton Res., 11, 165-170.

Goldman, J.C., 1980: Physiological processes, nutrient availability, and the concept of relative growth rate in marine phytoplankton ecology. In: Primary Productivity in the Sea. P.G. Falkowski, ed.. Plenum, New York. 179-194. , 1986: On phytoplankton growth rates and particulate C:N:P ratios at low light. Limnol. Oceanogr., 31, 13581363.
Greene, R.M., R.J. Geider and P.G. Falkowski, 1991: Effect of iron limitation on photosynthesis in a marine diatom. Limnol. Oceanogr., 36, 1772-1782.

Kiefer, D.A. and B.G. Mitchell, 1983: A simple, steady state description of phytoplankton growth based on absorption cross section and quantum efficiency. Limnol. Oceanogr., 28, 770-776.

and R.A. Reynolds, 1992: Advances in understanding phytoplankton fluorescence and photosynthesis. In: Primary Productivity and Biogeochemical $\mathrm{Cycles}$ in the Sea. P.G. Falkowski and A. Woodhead, eds., Plenum Press, New York, 155-174.

Kolber, Z., J. Zehr and P.G. Falkowski, 1988: Effects of growth irradiance and nitrogen limitation on photosynthetic energy conversion in photosystem II. Plant Physiol., $88,923-929$.

K.D. Wyman and P.G. Falkowski, 1990: Natural variability in photosynthetic energy conversion efficiency: a field study in the Gulf of Maine. Limnol. Oceanogr., 35, 72-79.

Laws, E.A., Gr.R. DiTullio and D.G. Redalje, 1987: High phytoplankton growth and production rates in the North Pacific subtropical gyre. Limnol. Oceanogr., 32, 905918.

Ley, A.C. and D. Mauzerall, 1982: Absolute absorption crosssections for photosystem II and the minimum quantum requirements for photosynthesis in Chlorella vulgaris. Biochim. Biophys. Acta, 680, 95-106.

Lorenzen, C.J., 1966: A method for the continuous measurement of in vivo chlorophyll concentration. Deep-Sea Res., 13, 223-227.

Martin, J.H., 1991: Iron, Liebig's Law and the greenhouse. Oceanography, 4, 52-55.

Mills, E.L., 1989: Biological Oceanography: An Early History, 1870-1960. Cornell University Press, Ithaca, 378 pp.

Morel, A., 1991: Light and marine photosynthesis: a spectral model with geochemical and climatological implications. Prog. Oceanogr., 26, 263-306.

Morris, I.. C.S. Yentsch and C.M. Yentsch, 1971: The physiological state with respect to nitrogen of phytoplankton from low-nutrient subtropical water as measured by the effect of ammonium ion on dark carbon dioxide fixation. Limnol. Oceanogr., 16, 859-868.

Platt, T., 1986: Primary production of the ocean water column as a function of surface light intensity: algorithms for remote sensing. Deep-Sea Res., 33, 149-163.

S. Sathyendranath, O. Ulloa, W.G. Harrison, N. Hoepffner and J. Goes, 1992: Nutrient control of phytoplankton photosynthesis in the Western North Atlantic. Nature, 356, 229-231.

Raven. J.A.. 1984: Energetics and Transport in Aquatic Plants. A.R. Liss, New York.

Reithman. H.C. and L.A. Sherman, 1988: Immunological characterization of iron-regulated membrane proteins in the cyanobacterium Anacystis nidulans R2. Plant Physiol., 88, 497-505.

Sverdrup, H.U., 1953: On conditions for the vernal blooming of phytoplankton. J. Cons. Explor. Mer., 18, 287-295.

Thomas, W.H.. 1970: On nitrogen deficiency in tropical Pacific Ocean phytoplankton: photosynthetic parameters in poor and rich water. Limnol. Oceanogr., 15, 380-385. and A. Dodson. 1972: On nitrogen deficiency in tropical Pacific Ocean phytoplankton. II. Photosynthetic and cellular characteristics of a chemostat-grown diatom. Limnol. Oceanogr., 17, 515-552.

Ward, B.B., 1990: Immunology in biological oceanography and marine ecology. Oceanography, 3, 30-35.

Yentsch, C.S., 1980: Phytoplankton growth in the sea-a coalescence of disciplines. In: Primary Productivity in the Sea. P.G. Falkowski. ed., Plenum, New York, 17-32.

Zehr, J.P., P.G. Falkowski, J. Fowler and D.G. Capone, 1988: Coupling between ammonium uptake and incorporation in a marine diatom: experiments with the shortlived radioisotope ${ }^{13}$ N. Limnol. Oceanogr. 33, 518527. $\square$ 